\title{
Local Approximation by Splines with Displacement of Nodes
}

\author{
V. T. Shevaldin ${ }^{1 *}$, E. V. Strelkova ${ }^{1}$, and Yu. S. Volkov ${ }^{2 * *}$ \\ ${ }^{1}$ Institute of Mathematics and Mechanics of the Ural Division of RAS, Yekaterinburg, 620990 Russia; \\ Ural Federal University, Yekaterinburg, 620002 Russia \\ ${ }^{2}$ Sobolev Institute of Mathematics, Novosibirsk, 630090 Russia \\ Received March 9, 2011
}

\begin{abstract}
We consider the problem of approximating a function defined on a uniform mesh by the method of local polynomial spline-approximation where the mesh of the nodes of the spline is chosen displaced relative to the mesh of the initial data. Conditions are established for the local form preservation by the spline of the initial data. We study the approximative properties of the method for the case of the simplest local approximation formula and find the optimal values of the displacement parameters.
\end{abstract}

DOI: $10.3103 / \mathrm{S} 1055134413010069$

Keywords: local spline-approximation, displaced data, Schoenberg approximation.

\section{INTRODUCTION}

Suppose that, in the nodes of a uniform mesh $\{j h\}_{j \in \mathbb{Z}}$ with step $h$ on the real axis, we are given the values $\left\{y_{j}\right\}_{j \in \mathbb{Z}}$ of some function $f(x), y_{j}=f(j h), j \in \mathbb{Z}$. Denote by $B(x)=B_{n, 0}(x)$ the normalized polynomial $B$-spline (of minimal defect) of degree $n$ with support $[0,(n+1) h]$ and nodes $0, h, \ldots,(n+$ 1) $h$ (for example, see [17]), and its displacement (shift) along the real axis $\alpha h(\alpha \in \mathbb{R})$ will be denoted by $B_{n, \alpha}(x)$, i.e., $B_{n, \alpha}(x)=B(x-\alpha h)$. norm

Denote by $L_{\infty}=L_{\infty}(\mathbb{R})$ the class of essentially bounded functions with the usual definition of the

$$
\|f\|_{\infty}=\operatorname{ess} \sup \{|f(x)|: x \in \mathbb{R}\}
$$

and denote by $W_{\infty}^{r}=\left\{f: f^{(r-1)} \in A C,\left\|f^{(r)}\right\|_{\infty} \leqslant 1\right\}(r \in \mathbb{N})$ the class of Sobolev functions.

It is known that the functions $\left\{B_{n, j}(x)\right\}_{j \in \mathbb{Z}}$ constitute a basis in the space of all polynomial splines on

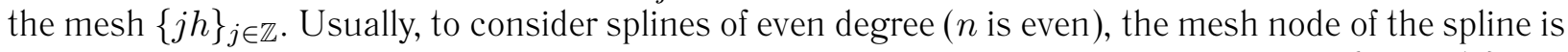
chosen displaced by one half step relative to the mesh of the nodes of the function; namely, $\{j h+h / 2\}_{j \in \mathbb{Z}}$, i.e., the system $\left\{B_{n, j+1 / 2}(x)\right\}_{j \in \mathbb{Z}}$ is chosen as a basis. Every spline $s(x)$ of degree $n$ can be written down as

$$
s(x)=\sum_{j} b_{j} B_{n, j-(n+1) / 2}(x) .
$$

A spline $s(x)$ is called local if the coefficients $b_{j}$ are defined by explicit formulas. They can also be determined from the interpolation conditions but, in this case, for their determination, one has to solve a system of equations. To approximate a given function $f(x)$ by a local spline, the coefficients $b_{j}$ are given by the values and derivatives of $f(x)$ in some small neighborhood of the node $j h$, for example, in the form of some functionals which can be written as linear combinations of the values of $f(x)$ in the mesh nodes.

\footnotetext{
${ }^{*}$ E-mail: valerii.shevaldin@imm.uran.ru

${ }^{* *}$ E-mail: volkov@math.nsc.ru
} 
Local approximation methods have become a standard tool in approximation theory and numerical analysis, mainly as a useful alternative to interpolation. Their main advantage is that, unlike in interpolation, there is no need to solve any system of equations. Moreover, the use of local approximation does not necessarily lead to a loss of accuracy compared with interpolation. There are schemes of local approximation in which, as in the case of interpolation, the maximal order of accuracy is attained. These are often called quasi-interpolation.

Well known is the simplest local approximation formula

$$
s(x)=\sum_{j} y_{j} B_{n, j-(n+1) / 2}(x)
$$

which approximates a smooth function $f(x)$ up to $O\left(h^{2}\right)$. In general, (nonuniform mesh), the formula has only first approximation order but, on a uniform mesh, it coincides with the Schoenberg approximation [10] which diminishes variation and always has second order. The operator Schoenberg operator is a positive operator, and so it can only approximate smooth functions with at most second order [4]. But the property of variation-diminishing guarantees the inheritance by the Schoenberg spline of such properties as positivity, monotonicity, and convexity, in their presence for the approximated function $f(x)$. Even low-degree interpolation splines in general do not preserve such geometric properties. Simple sufficient conditions for form preservation (preservation of sign-definiteness of some derivative) with interpolation by cubic splines, are established in [15].

The simplest formula (1) for $n=2$ was studied in detail in [14] (for a nonuniform mesh, in [11]). It is interesting to note that, in the periodic case on parabolic splines, this simple scheme implements the Kolmogorov width and the relative width of the function class $W_{\infty}^{2}$ [14], both for the function and for the derivative. The simplest formula (1) for cubic splines $(n=3)$ gives a greater constant in the estimation of the approximation error compared with $n=2(1 / 6$ instead of $1 / 8)$, but, in the estimate for the approximation of the derivative, the constant $1 / 2$ is preserved [17] (including for a nonuniform mesh [9]).

De Boor and Fix showed in [1] that if, in (1), the values $y_{j}$ are replaced by some functionals $\lambda_{j} f$ calculated not only via the values of $f(x)$ but also by means of the values of its derivatives then this expression approximates $f(x)$ with the same maximal order $O\left(h^{n+1}\right)$ as an interpolation spline of the same degree (this property explains the meaning of the term quasi-interpolation). Later Lyche and Schumaker showed in [6] that the maximal order may be attained using only the values of $f(x)$ (without derivatives). Both these local approximations are accurate (see $[1,6]$ ) in the space $\mathbb{P}_{n}$ of the polynomials of degree $n$, i.e., the resulting spline $s(x)$ reproduces a function $f(x)$ that is a polynomial of degree $n$. The simplest formula (1) is accurate only on $\mathbb{P}_{1}$. It is established (for example, see [17]) that if in the formula

$$
s(x)=\sum_{j} I_{j} B_{n, j-(n+1) / 2}(x),
$$

where $I_{j}=\sum_{m \in M} c_{m} y_{j+m}$ and $M$ is a set of integer indices, the coefficients $c_{m}$ are chosen from the condition of the accuracy of the formula on $\mathbb{P}_{k}(k \leqslant n)$ then $s(x)$ approximates any sufficiently smooth function $f(x)$ with order $O\left(h^{k+1}\right)$.

Of course, of the greatest interest are the approximations accurate on $\mathbb{P}_{n}$ having the maximal accuracy order $O\left(h^{n+1}\right)$. Explicit formulas for these approximations are written down for small $n$. The case of cubic splines $n=3$ (in general, for nonuniform meshes) is considered in detail in [17] and for parabolic splines $(n=2)$ in [5]. In [3], Korne ̌chuk found accurate estimates for the approximation of some classes of differentiable functions.

In this article, we consider a local approximation of the form

$$
s(x)=\sum_{j} I_{j} B_{n, j+\alpha}(x),
$$

where

$$
I_{j}=\sum_{m \in M} c_{m} y_{j+m}
$$


$M$ is a set of integers, and the node mesh of the spline is displaced by $\alpha h$ relative to the mesh of the known values of the approximated function $f(x)$. For splines of an arbitrary odd degree, only the case $\alpha=0$ has been considered, and for cubic splines, Strelkova in [12] studied the approximation for $\alpha=1 / 2$. For splines of even degree, only the case $\alpha=1 / 2$ was studied, and for parabolic splines $(n=2)$, the case of arbitrary $\alpha$ (see [13]). In Section 2, we study the form-preserving properties of a local approximation method of the form (3) and (4); here we establish conditions of the $k$-monotonicity of the local spline. In Section 3, we study the approximate properties of the simplest formula $\left(I_{j}=y_{j}\right)$ of local approximation with displacement $\alpha h$ and find an optimal value of the displacement parameter $\alpha$ that guarantees the maximal possible approximation order for the simplest formula.

\section{FORM-PRESERVING PROPERTIES OF LOCAL APPROXIMATION}

We consider local approximation of the form (3) and (4). Since this is a local approximation, the set $M$ should contain a small number of indices. Denote by $m_{0}$ and $m_{1}$ the smallest and greatest indices in $M$, respectively. We may assume that $M$ contains all the integers from $m_{0}$ to $m_{1}$ by putting $c_{m}=0$ for $m_{0}<m<m_{1}$ not contained in $M$. We are interested in the possibility of the preservation of the (local) geometric characteristics of the approximated function $f(x)$, i.e., of the preservation of $k$-monotonicity. Explain what is meant by the preservation of $k$-monotonicity.

Let $\Delta^{k} y_{j}=\sum_{s=0}^{k}(-1)^{k-s} C_{k}^{s} y_{j+s}$ be the finite difference of order $k$ of the given values of the function of $y_{j}=f(j h)$ with step $h$ (here $C_{k}^{s}$ are the binomial coefficients). If for some $k(k \leqslant n)$, all the finite differences $\Delta^{k} y_{j}$ of order $k$ (for $k=0$, these are the values $y_{j}$ themselves) are nonnegative then the corresponding derivative of the spline $s(x)$ must be nonnegative too; i.e., $s^{(k)}(x) \geqslant 0$. Under the local preservation of $k$-monotonicity we understand the nonnegativity of the function $s^{(k)}(x)$ on some interval of the mesh because of the nonnegativity of some number of finite differences $\Delta^{k} y_{j}$ close to the interval under consideration. Note that, for small values of $k, k$-monotonicity has special names: $k=0-$ nonnegativity, $k=1-$ monotonicity, $k=2-$ convexity.

Theorem 1. Suppose that $c_{m} \geqslant 0, m \in M$. A spline of the form (3) and (4) inherits the properties of the $k$-monotonicity of the initial data $\left\{y_{j}\right\}$; namely:

1) if $y_{j} \geqslant 0$ for $j=m_{0}-n+l, \ldots, m_{1}+l$ then $s(x) \geqslant 0$ for $x \in[(l+\alpha) h,(l+1+\alpha) h]$;

2) if $\Delta^{k} y_{j} \geqslant 0(1 \leqslant k \leqslant n)$ for $j=m_{0}-n+l, \ldots, m_{1}+l-k$ then $s^{(k)}(x) \geqslant 0$ for $x \in[(l+$ $\alpha) h,(l+1+\alpha) h]$.

Proof. Let $x \in[(l+\alpha) h,(l+1+\alpha) h]$ for some $l \in \mathbb{Z}$. Then the sum in (3) contains finitely many summands

$$
s(x)=\sum_{j=l-n}^{l} I_{j} B_{n, j+\alpha}(x) .
$$

Since $I_{j}=c_{m_{0}} y_{j+m_{0}}+\cdots+c_{m_{1}} y_{j+m_{1}}$, the sum (5) contains the initial data from $y_{l-n+m_{0}}$ to $y_{l+m_{1}}$. Consequently, if the values $y_{l-n+m_{0}}, \ldots, y_{l+m_{1}}$ are nonnegative then so are $I_{l-n}, \ldots, I_{l}$. Thus, if $x \in$ $[(l+\alpha) h,(l+1+\alpha) h]$ then $s(x) \geqslant 0$; the first part of the theorem is proven.

The second part follows from similar arguments and the following two lemmas.

Lemma 1. $\Delta^{k} I_{j}=c_{m_{0}} \Delta^{k} y_{j+m_{0}}+\cdots+c_{m_{1}} \Delta^{k} y_{j+m_{1}}$.

Lemma 2. $s^{(k)}(x)=h^{-k} \sum_{j=l-n}^{l-k} \Delta^{k} I_{j} B_{n-k, j+k+\alpha}(x)$.

Proof of Lemma 1 immediately follows from the definition of the finite difference and the representation (4), and the proof of Lemma 2 results from the formula for the derivative of a spline expanded in $B$ splines ( see [17]).

The conditions $c_{m} \geqslant 0, m \in M$, in Theorem 1 are not only sufficient for the preservation of $k$ monotonicity for each $k \leqslant n$ but also necessary on the class of $k$-monotone functions. It is clear that these conditions define a positive linear approximation method (3) and (4). This means (see [4]) that 
the approximation order by the local splines (3) and (4) provided that $c_{m} \geqslant 0, m \in M$, of functions differentiable at least twice can not be greater than $h^{2}$. Thus, the use of a local approximation accurate on the space of the polynomials of degree at least one, i.e., $\mathbb{P}_{k}(k>1)$, is possible only if the coefficients $c_{m}$ in (4) have different signs. This means that the preservation of $k$-monotonicity does not hold for every approximated $k$ - monotone function.

Note that the simplest local approximation formula $\left(I_{j}=y_{j}\right)$ always preserves the $k$-monotonicity of the initial data. In the classical case $\left(\alpha=-\frac{n+1}{2}\right)$, such a scheme coincides with the Schoenberg variation-diminishing approximation for a uniform mesh. It is known (see [7]) that, in the case of an arbitrary mesh, the Schoenberg spline preserves $k$-monotonicity for $k=0,1,2$ but does not for $k=3$. As far as we know, the question of the preservation of $k$-monotonicity for $k \geqslant 3$ has not been considered for a uniform mesh before.

\section{THE SIMPLEST LOCAL APPROXIMATION FORMULA}

In this section, we study the approximate properties of the simplest local approximation formula $\left(I_{j}=y_{j}\right)$ with displacement $\alpha h$ and find out the influence of $\alpha$ on the accuracy of the formula.

It is known (see [7]) that the simplest formula on a uniform mesh reconstructs degree-one polynomials. Though the functions $x^{2}$ are no longer reconstructed by the simplest formula, as follows from Marsden's Identity (see [7]), if $\left\{t_{j}\right\}$ is the mesh of the nodes of a spline of degree $n$ then the functions $x^{k}$ $(k \leqslant n)$ can be expanded in $B$-splines; in particular, the following formulas hold:

where

$$
x=\sum_{j} t_{j}^{*} B_{n, j+\alpha}(x), \quad x^{2}=\sum_{j} t_{j}^{* *} B_{n, j+\alpha}(x),
$$

$$
\begin{gathered}
t_{j}^{*}=\left(t_{j+1}+\cdots+t_{j+n}\right) / C_{n}^{1}, \\
t_{j}^{* *}=\left(t_{j+1} t_{j+2}+t_{j+1} t_{j+3}+\cdots+t_{j+n-1} t_{j+n}\right) / C_{n}^{2} .
\end{gathered}
$$

In our case,

$$
t_{j}^{*}=\left(j+\alpha+\frac{n+1}{2}\right) h, \quad t_{j}^{* *}=\left(t_{j}^{*}\right)^{2}-\frac{n+1}{12} h^{2} .
$$

The following two lemmas are valid.

Lemma $3 . \sum_{j}(j h-x) B_{n, j+\alpha}(x)=-\left(\alpha+\frac{n+1}{2}\right) h$.

Lemma 4. $\sum_{j}(j h-x)^{2} B_{n, j+\alpha}(x)=\left(\alpha+\frac{n+1}{2}\right)^{2} h^{2}+\frac{n+1}{12} h^{2}$ for $n \geqslant 2$.

The expressions on the right-hand sides in Lemmas 3 and 4 are independent of $x$, but for splines of degree one $(n=1)$, the expression obtained for the sum on the left-hand side of 4 already depends on $x$; namely, if $x=(l+\alpha+t) h, 0 \leqslant t \leqslant 1$, then we have the equality

$$
\sum_{j}(j h-x)^{2} B_{1, j+\alpha}(x)=(\alpha+1)^{2} h^{2}+t(1-t) h^{2} .
$$

Now, Lemmas 3 and 4 enable us to get the expansion of the spline

$$
s(x)=s_{n, \alpha}(f, x)=\sum_{j} y_{j} B_{n, j+\alpha}(x)
$$

by Taylor's formula at the point $x$ for a function $f \in C^{r}(r \geqslant 3)$. Take

$$
x \in[(l+\alpha) h,(l+1+\alpha) h] .
$$


Since

$$
y_{j}=f(j h)=f(x)+f^{\prime}(x)(j h-x)+\frac{1}{2} f^{\prime \prime}(x)(j h-x)^{2}+O\left(h^{3}\right),
$$

we have

$$
\begin{aligned}
s_{n, \alpha}(f, x)=f(x) & +f^{\prime}(x) \sum_{j=l-n}^{l}(j h-x) B_{n, j+\alpha}(x) \\
& +\frac{1}{2} f^{\prime \prime}(x) \sum_{j=l-n}^{l}(j h-x)^{2} B_{n, j+\alpha}(x)+O\left(h^{3}\right) .
\end{aligned}
$$

After easy transformations, for $n \geqslant 2$, we infer by Lemmas 3 and 4 that

$$
\begin{aligned}
s_{n, \alpha}(f, x)=f(x)-f^{\prime}(x) h\left[\alpha+\frac{n+1}{2}\right] \\
+\frac{1}{2} f^{\prime \prime}(x) h^{2}\left[\left(\alpha+\frac{n+1}{2}\right)^{2}+\frac{n+1}{12}\right]+O\left(h^{3}\right) .
\end{aligned}
$$

As follows from (6), for $n=1$, instead of the value $\frac{n+1}{12}$, this equality contains the expression $t(1-t)$.

We see from expansion (7) that, for any value of the displacement parameter $\alpha$, the simplest local approximation formula approximates the initial function with the first order, and only $\alpha=-\frac{n+1}{2}$ yields an increase of the order of approximation. The approximation order becomes equal to $h^{2}$, and this is the best value of $\alpha$. Note that, for $\alpha=-\frac{n+1}{2}$, we obtain the classical simple local approximation formula in which the value $y_{j}$ is taken in the middle of the support of the corresponding $B$-spline $B_{n, j+\alpha}(x)$. Therefore, we have:

Theorem 2. If $f \in C^{r}, r \geqslant 3$, then the estimate

$$
\left\|s_{n, \alpha}-f\right\|_{\infty} \leqslant \frac{n+1}{24} h^{2}\left\|f^{\prime \prime}\right\|_{\infty}+O\left(h^{3}\right) .
$$

holds for $\alpha=-\frac{n+1}{2}(n \geqslant 2)$.

An analogous estimate is valid also for splines of degree one but the constant in (8) is somewhat larger; namely, it is the same as for $n=2$ (see [17]), i.e.,

$$
\left\|s_{1,-1}-f\right\|_{\infty} \leqslant \frac{1}{8} h^{2}\left\|f^{\prime \prime}\right\|_{\infty}+O\left(h^{3}\right) .
$$

Observe that estimate (8) of Theorem 2 was established in 1965 by Schoenberg [10]. We now prove that we can discard the terms of order $O\left(h^{3}\right)$ in (8).

Theorem 3. If $\alpha=-\frac{n+1}{2}(n \geqslant 2)$ then

$$
\sup _{f \in W_{\infty}^{2}}\left\|s_{n, \alpha}-f\right\|_{\infty}=\frac{n+1}{24} h^{2} .
$$

Proof. Suppose that $\alpha=-\frac{n+1}{2}, x \in\left[\left(l-\frac{n+1}{2}\right) h,\left(l+1-\frac{n+1}{2}\right) h\right]$. Then

$$
\left|s_{n, \alpha}(f, x)-f(x)\right|=\left|\sum_{j=l-n}^{l} B_{n, j-(n+1) / 2}(x) \int_{x}^{j h}(j h-\tau) f^{\prime \prime}(\tau) d \tau\right| .
$$

Since the factors at $f^{\prime \prime}(\tau)$ are nonnegative in each summand, we have 


$$
\begin{aligned}
\left|s_{n, \alpha}(f, x)-f(x)\right| & \leqslant\left(\sum_{j=l-n}^{l} B_{n, j-(n+1) / 2}(x) \int_{x}^{j h}(j h-\tau) d \tau\right)\left\|f^{\prime \prime}\right\|_{\infty} \\
& =\left[\sum_{j=l-n}^{l} \frac{1}{2}(j h-x)^{2} B_{n, j-(n+1) / 2}(x)\right]\left\|f^{\prime \prime}\right\|_{\infty} \\
& =\frac{n+1}{24} h^{2}\left\|f^{\prime \prime}\right\|_{\infty},
\end{aligned}
$$

with equality attained for $f(x)=x^{2} / 2$.

Note that, for parabolic splines ( $n=2$ ), Theorem 3 was proved by Subbotin [14] who showed that, in the periodic case, this method realizes both Kolmogorov and Konovalov widths. Estimate (9) of Theorem 3 is well known also for cubic splines (see [17]).

For splines of arbitrary degree (the Schoenberg splines), apart from estimates in the class of continuous functions ( see [7, 8, 10]), there are known estimates in $L_{1}$ with extension to $L_{p}$ [2].

The present article is an exposition of a part of the talk at the Russian Conference "Methods of Spline-Functions" dedicated to the 80th anniversary of Yu. S. Zavyalov (see [16]).

\section{ACKNOWLEDGMENTS}

The work was partially supported by Joint Integration Projects of the Siberian Division of RAS (grant 2009-14) and the Ural Division of RAS (grant 09-C-1-1007) and by the Russian Foundation for Basic Research (grant 11-07-00447).

\section{REFERENCES}

1. C. de Boor C. and G. J Fix, "Spline approximation by quasiinterpolants," J. Approximation Theory 8 (1) $19-45$ (1973).

2. W. Hoeffding, "The $L_{1}$ norm of the approximation error for splines with equidistant knots," J. Approximation Theory 11 (2), 176-193(1974).

3. N. P. Korne chuk, Splines in Approximation Theory (Nauka, Moscow, 1984) [in Russian].

4. P. P. Korovkin, Linear Operators and Approximation Theory (GIFML, Moscow, 1959) [Russian Monographs and Texts on Advanced Mathematics and Physics, Vol. III (Gordon and Breach Publishers, New York; Hindustan Publishing Corp. Delhi; 1960)].

5. B. I. Kvasov, "Parabolic B-splines in interpolation problems," Zh. Vychisl. Mat. Mat. Fiz. 23 (2), 278-289 (1983) [U.S.S.R. Comput. Math. Math. Phys 23 (2), 13-19 (1983)].

6. T. Lyche and L. L. Schumake, "Local spline approximation methods," J. Approximation Theory 15 (4), 294325 (1975).

7. M. J. Marsden, "An identity for spline functions with applications to variation-diminishing spline approximation," J. Approximation Theory 3 (1), 7-49 (1970).

8. M. J. Marsden and I. J. Schoenberg I. J., "On variation diminishing spline approximation methods," Mathematica $C l u j, 8$ (31) (1), 61-82(1966).

9. T. E. Ovchinnikova, "Sharp error estimates for approximation of local approximation by cubic splines. A formula that is exact on first-degree polynomials," Vychisl. Sistemy, No. 128, 39-59 (1988) [in Russian].

10. I. J. Schoenberg, "On spline functions," Inequalities (Proc. Sympos. Wright-Patterson Air Force Base, Ohio, 1965), 255-291 (Academic Press, New York, 1967).

11. V. T. Shevaldin, "Approximation by local parabolic splines with arbitrary knots," Sib. Zh. Vychisl. Mat. 8(1), 77-88 (2005) [in Russian].

12. E. V. Shevaldina, "Inheritance of $k$-monotonicity under approximation by local cubic splines," Proceedings of the 40th All-Russia Youth Conference "Modern Problems of Mathematics," 106-110 (Ural Division of RAS, Yekaterinburg, 2009) [in Russian]. 
13. E. V. Strelkova and V. T. Shevaldin, "A method of approximation by local parabolic splines," Abstracts of the 42th All-Russia Youth Conference "Modern Problems of Mathematics," pp. 148-150 (Ural Division of RAS, Yekaterinburg, 2011) [in Russian]

14. Yu. N. Subbotin, "Inheritance of monotonicity and convexity properties in local approximation," Zh. Vychisl. Mat. Mat. Fiz. 33 (7), 996-1003, (1993) [Comput. Math. Math. Phys. 33 (7), 879-884 (1993)].

15. Yu. S. Volkov, V. V. Bogdanov, V. L. Miroshnichenko, and V. T. Shevaldin, "Shape-preserving interpolation by cubic splines," Mat. Zametki 88 (6), 836-844 (2010) [Math. Notes 88 (5-6), 798-805 (2010)].

16. Yu. S. Volkov, E. T. Strelkova, and V. T. Shevaldin, "On local approximation by cubic splines," The Russian Conference "Methods of Spline-Functions" Dedicated to the 80-th Anniversary of Yu. S. Zavyalov. Abstracts, pp. 35-36 (Sobolev Institute of Mathematics, Novosibirsk, 2011) [in Russian].

17. Yu. S. Zavyalov, B. I. Kvasov, and V. L. Miroshnichenko, Methods of Spline-Functions (Nauka, Moscow, 1980) [in Russian]. 